\title{
Hepatitis $B$ reactivation in patients receiving immunosuppressive therapy: a hidden menace
}

\author{
Chih-Lin Lin ${ }^{1,2} \cdot$ Jia-Horng Kao ${ }^{3,4,5,6}$
}

Received: 22 November 2016/ Accepted: 29 December 2016/Published online: 5 January 2017

(C) Asian Pacific Association for the Study of the Liver 2017

Hepatitis B virus (HBV) infection is prevalent in Asia, Africa, Southern Europe, and Latin America, where the rate of chronic HBV infection in the general population ranges from 2 to $20 \%$ [1]. Acute flares in the natural course of chronic HBV infection are not uncommon. The pathogenesis of acute flares is related to interactions between the virus and host immune responses to HBVencoded antigens [2]. Therefore, patients who have chronic HBV infection are vulnerable to surges of viral replication and widespread infection of hepatocytes during immunosuppressive therapy or chemotherapy [3] Subsequently, immune reconstitution against HBV-infected hepatocytes after stopping immunosuppressive therapy or chemotherapy will lead to liver cell damage and hepatitis flare [4]. HBV reactivation has been increasingly recognized in chronic hepatitis B (CHB) patients with hematological malignancies, hematopoietic

Jia-Horng Kao

kaojh@ntu.edu.tw

1 Department of Gastroenterology, Renai Branch, Taipei City Hospital, Taipei, Taiwan

2 Department of Psychology, National Chengchi University, Taipei, Taiwan

3 Graduate Institute of Clinical Medicine, National Taiwan University College of Medicine, 1 Chang-Te St., Taipei 10002, Taiwan

4 Department of Internal Medicine, National Taiwan University Hospital, Taipei, Taiwan

5 Hepatitis Research Center, National Taiwan University, National Taiwan University Hospital, Taipei, Taiwan

6 Department of Medical Research, National Taiwan University, National Taiwan University Hospital, Taipei, Taiwan stem cell transplantation (HSCT), as well as solid tumor following chemotherapy [5]. The incidence rate of HBV reactivation ranges between 14 and $72 \%$, depending on malignancy type, immunosuppressive agents used, and hepatitis B status [3]. Although HBV reactivation commonly occurs in cancer patients who receive chemotherapy, it can also occur in patients with nonmalignant diseases, such as rheumatoid arthritis and inflammatory bowel disease, due to use of immunosuppressive therapy or biological agents $[6,7]$. The clinical manifestations of HBV reactivation in patients receiving immunosuppressive agents vary from asymptomatic hepatitis flare to fatal liver failure. Furthermore, anticancer therapy may be interrupted due to hepatitis flares, thus affecting the prognosis of cancer patients. There is ample evidence showing that prophylactic antiviral therapy is effective to avoid the risk of HBV reactivation [8]. In several international guidelines for management of HBV infection, prophylaxis with antiviral agents before starting chemotherapy or immunosuppressive agent is strongly recommended [9-12]. However, patients who have resolved or so-called occult HBV infection (OBI), as defined by presence of HBV DNA in liver or serum as well as HBsAg negativity/anti-HBc positivity with or without anti-HBs, also carry high risk of HBV reactivation when their host immunity is severely compromised by chemotherapy or immunosuppressive therapy [13].

In this issue, the incidence rate of $\mathrm{HBV}$ reactivation in patients undergoing allogeneic HSCT, including CHB patients with low replication and patients with resolved HBV infection, and possible factors associated with HBV reactivation are discussed by Jun et al. [14], who retrospectively analyzed 506 patients who underwent allogenic/ autologous HSCT. Overall, the rate of HBV reactivation, defined by tenfold increase in HBV DNA level compared 
with baseline or HBV DNA detection in a patient with negative HBV DNA before HSCT, was $4.2 \%$. Although most HBsAg-positive patients received preemptive antiviral treatment, the HBV reactivation rate among $\mathrm{HBsAg-}$ positive patients was much higher than that among HBsAgnegative/anti-HBc-positive patients (14.3 versus 5.8\%). Of particular note, the incidence rate of HBV reactivation in patients with resolved HBV infection was not low (5.9\%). These findings confirm that patients with resolved or OBI still have high risk of HBV reactivation. However, several issues are worthy of discussion. First, in this study, anti$\mathrm{HBc}$ was not checked in $35.4 \%$ of the HBsAg-negative HSC donors. Like a recent survey of American Association for the Study of Liver Disease (AASLD) members regarding HBV reactivation during cancer chemotherapy, HBV screening before chemotherapy was performed in $53 \%$ of cases, and only $10 \%$ of CHB patients received prophylactic antiviral therapy [15]. The clinical challenge is thus how to reinforce physicians' awareness of the need to screen for HBV infection and identify patients at risk of HBV reactivation. Therefore, all subjects, including recipients and donors, should receive $\mathrm{HBV}$ serologic screening before immunosuppressive therapy. Screening should include assessment of HBsAg and anti-HBc. In $\mathrm{HBV}$-endemic regions, the seroprevalence rate of anti-HBc is extremely high; for example, the overall prevalence of anti-HBc was $68.46 \%$ in a population-based survey in Taiwan [16]. The risk of HBV reactivation was increased in HBsAg-negative/anti-HBc-positive patients with detectable serum HBV DNA, especially in patients who received chemotherapy in conjunction with rituximab [3]. In a prospective study of 150 lymphoma patients with resolved HBV infection who received rituximab-based chemotherapy, the incidence rate of HBV reactivation and HBV-related hepatitis flare was 10.4 and 6.4 per 100 person-years, respectively. Patients with hepatitis flare exhibited significantly higher frequency of reappearance of HBsAg after HBV reactivation (100 versus 28.5\%; $p=0.003$ ) [17]. In addition, the role of antiviral prophylaxis in preventing HBV reactivation before rituximabbased chemotherapy in lymphoma patients with resolved hepatitis B was demonstrated in a randomized controlled trial in Taiwan. Antiviral prophylactic therapy significantly decreased the rate of HBV reactivation [18]. Above all, establishment of computerized physician order entry-based alert and therapeutic control systems in hospitals is mandatory to improve HBV screening and prophylaxis in CHB patients before immunosuppressive therapy or chemotherapy [19]. Taken together, accumulating evidence strongly recommends that all patients should be screened for evidence of HBV exposure prior to chemotherapy or immunosuppressive treatment. Patients with positive HBsAg should receive prophylactic antiviral therapy, whereas patients with positive anti-HBc alone should consider prophylactic antiviral therapy.

The second issue is to identify predictors of HBV reactivation for patients receiving immunosuppressive treatment. In the current study, old age ( $\geq 60$ years) and HBsAg-positive donor were significant risk factors of HBV reactivation in patients who underwent HSCT. With recent advances in molecular investigations, serum biomarkers associated with the natural history of chronic HBV infection have been identified. Integration of these biomarkers may improve prediction of HBV reactivation for patients receiving immunosuppressive treatment [20, 21]. An association between serum anti-HBsAg level and HBV reactivation in patients with resolved hepatitis $B$ and receiving rituximab-containing chemotherapy has been suggested. The 2-year cumulative rate of HBV reactivation was $41.5 \%$. At baseline, patients without anti-HBs, compared with those with positive anti-HBs, had a significantly higher rate of HBV reactivation $(68.3$ versus $34.4 \% ; p=0.012$ ). Baseline undetectable anti-HBs was the significant risk factor associated with HBV reactivation [hazard ratio $3.51 ; 95 \%$ confidence interval (CI) 1.37-8.98; $p=0.009$ ] [22]. Hepatitis B virus core-related antigen ( $\mathrm{HBcrAg}$ ) is a novel serological marker, comprising hepatitis $\mathrm{B}$ core antigen ( $\mathrm{HBcAg}$ ), $\mathrm{HBeAg}$, and a precore protein (p22crAg) [23]. Both $\mathrm{HBcAg}$ and $\mathrm{HBeAg}$, sharing an identical amino acid sequence, are targets of cytotoxic $\mathrm{T}$ cells, thus HBcrAg can induce host cellular immune responses. Recently, the correlation of serum HBcrAg with HBV reactivation among patients with resolved hepatitis $B$ undergoing immunosuppressive therapy was investigated in a prospective study [24]. Baseline HBcrAg-positive patients had a significantly higher 2-year HBV reactivation rate than $\mathrm{HBcrAg}$-negative patients (71.8 versus $31 \%, p=0.002$; hazard ratio $2.94,95 \% \mathrm{CI}$ $1.43-6.07, p=0.004)$. Therefore, HBcrAg can serve as a predictor for identification of patients who would benefit from prophylactic antiviral treatment.

Host genetic variations also contribute to immunosuppressive agent-related HBV reactivation. A genetic association study in a cohort of HBsAg-negative, non-Hodgkin lymphoma patients in Taiwan demonstrated that the interleukin 4 haplotype rs2243248-rs2243263 was significantly associated with $\mathrm{HBV}$ reactivation and $\mathrm{HBsAg}$ reverse seroconversion, regardless of anti-HBs status [25]. Therefore, host genetic background should be taken into consideration to identify resolved HBV patients with risk of $\mathrm{HBV}$ reactivation.

In summary, patients with inactive or even resolved HBV infection undergoing immunosuppressive therapy are at high risk of HBV reactivation. However, physicians' awareness in terms of screening patients who are at risk of HBV reactivation remains unsatisfactory. Strategies to 


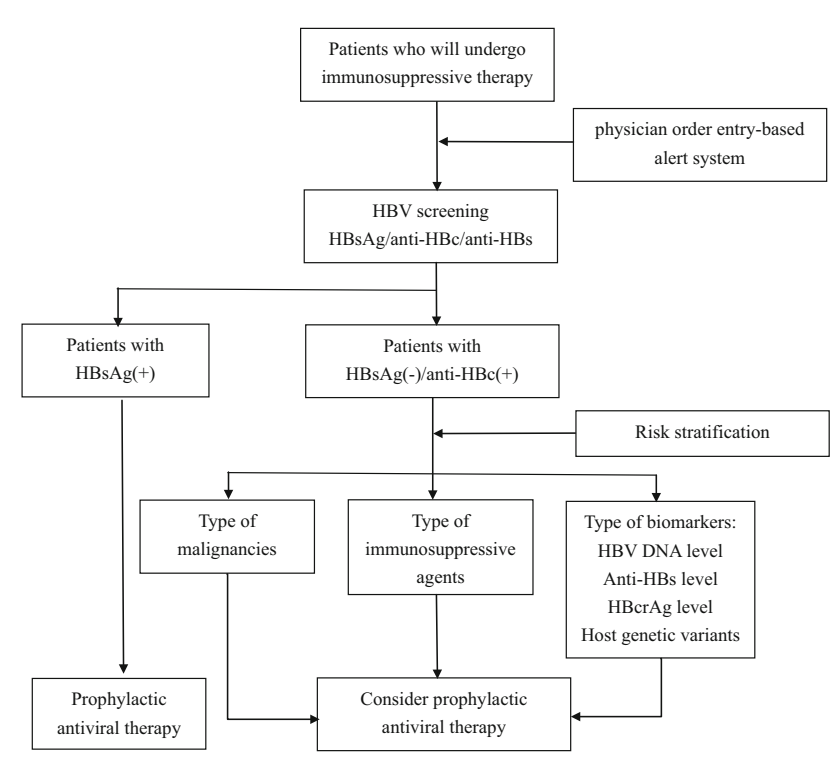

Fig. 1 Proposed algorithm for screening and treatment of patients receiving immunosuppressive therapy. $H B V$ hepatitis $\mathrm{B}$ virus, $H B s A g$ hepatitis B surface antigen, $H B c$ hepatitis B core antigen, $H B c r A g$ hepatitis B virus core-related antigen

overcome this hidden menace include exploration of predictors associated with HBV reactivation and implementation of optimal physician order entry-based alert screening and prophylactic therapeutic control systems among patients at risk of $\mathrm{HBV}$ reactivation (Fig. 1). Implementation of these control measures for risk stratification and prophylactic antiviral therapy could render HBV reactivation a preventable disease.

\section{Compliance with ethical standards}

Conflict of interest Chih-Lin Lin and Jia-Horng Kao have no conflict of interest.

Ethical approval This article does not contain any studies with human participants or animals performed by any of the authors.

\section{References}

1. Kao JH, Chen DS. Global control of hepatitis B virus infection. Lancet Infect Dis 2002;2:395-403

2. Lok ASF. Hepatitis B infection: pathogenesis and management. J Hepatol 2000;32(suppl. 1):89-97

3. Liu CJ, Chen PJ, Chen DS, Kao JH. Hepatitis B virus reactivation in patients receiving cancer chemotherapy: natural history, pathogenesis, and management. Hepatol Int 2013;7:316-326

4. Vento S, Cainelli F, Longhi MS. Reactivation of replication of hepatitis $\mathrm{B}$ and $\mathrm{C}$ viruses after immunosuppressive therapy: an unresolved issue. Lancet Oncol 2002;3:333-340

5. Yeo W, Johnson PJ. Diagnosis, prevention and management of hepatitis $\mathrm{B}$ virus reactivation during anticancer therapy. Hepatology 2006;43:209-220

6. Gwak GY, Koh KC, Kim HY. Fatal hepatic failure associated with hepatitis B virus reactivation in a hepatitis B surface antigen-negative patient with rheumatoid arthritis receiving low dose methotrexate. Clin Exp Rheumatol 2007;25:888-889
7. Loras C, Gisbert JP, Mínguez M, et al. Liver dysfunction related to hepatitis $\mathrm{B}$ and $\mathrm{C}$ in patients with inflammatory bowel disease treated with immunosuppressive therapy. Gut 2010;59:1340-1346

8. Loomba R, Rowley A, Wesley R, et al. Systematic review: the effect of preventive lamivudine on hepatitis $\mathrm{B}$ reactivation during chemotherapy. Ann Intern Med 2008;148:519-528

9. Lok AS, McMahon BJ. Chronic hepatitis B: update 2009. Hepatology 2009;50:661-662

10. European Association For The Study Of The Liver. EASL Clinical Practice Guidelines: management of chronic hepatitis B virus infection. J Hepatol 2012;57:167-185

11. Sarin SK, Kumar M, Lau GK, et al. APASL clinical practice guidelines on the management of hepatitis B: a 2015 update. Hepatol Int. 2016;10:1-98

12. WHO. Guidelines for the prevention, care and treatment of persons with chronic hepatitis B infection. Geneva Switzerland: World Health Organization; 2015

13. Lin CL, Kao JH. Perspectives and control of hepatitis B virus infection in Taiwan. J Formos Med Assoc 2015;114:901-909

14. Jun $\mathrm{CH}$, Kim BS, Oak $\mathrm{CY}$, et al. $\mathrm{HBV}$ reactivation risk factors in patients with chronic HBV infection with low replicative state and resolved HBV infection undergoing hematopoietic stem cell transplantation in Korea. Hepatol Int 2016 (Epub ahead of print)

15. Hwang JP, Barbo AG, Perrillo RP. Hepatitis B reactivation during cancer chemotherapy: an international survey of the membership of the American Association for the Study of Liver Diseases. J Viral Hepat 2015;22:346-352

16. Chen CL, Yang JY, Lin SF, Sun CA, Bai CH, You SL, et al. Slow decline of hepatitis B burden in general population: Results from a population-based survey and longitudinal follow-up study in Taiwan. J Hepatol 2015;63:354-363

17. Hsu C, Tsou HH, Lin SJ, Wang MC, Yao M, Hwang WL, et al. Taiwan Cooperative Oncology Group. Chemotherapy-induced hepatitis B reactivation in lymphoma patients with resolved HBV infection: a prospective study. Hepatology 2014;59:2092-2100

18. Huang YH, Hsiao LT, Hong YC, Chiou TJ, Yu YB, Gau JP, et al. Randomized controlled trial of entecavir prophylaxis for rituximab-associated hepatitis B virus reactivation in patients with lymphoma and resolved hepatitis B. J Clin Oncol 2013;31:2765-2772

19. Hsu PI, Lai KH, Cheng JS, et al. Prevention of acute exacerbation of chronic hepatitis B infection in cancer patients receiving chemotherapy in a hepatitis B virus endemic area. Hepatology 2015;62:387-396

20. Lin CL, Kao JH. New perspectives of biomarkers for the management of chronic hepatitis B. Clin Mol Hepatol. 2016;22:423-31

21. Yang HC, Kao JH. Looking into the crystal ball: biomarkers for outcomes of HBV infection. Hepatol Int 2016;10:99-101

22. Seto WK, Chan TS, Hwang YY, et al. Hepatitis B reactivation in patients with previous hepatitis B virus exposure undergoing rituximab-containing chemotherapy for lymphoma: a prospective study. J Clin Oncol 2014;20;32:3736-3743

23. Kimura T, Rokuhara A, Sakamoto Y, et al. Sensitive enzyme immunoassay for hepatitis B virus core-related antigens and their correlation to virus load. J Clin Microbiol 2002;40:439-445

24. Seto WK, Wong DH, Chan TY, et al. Association of hepatitis B core-related antigen with hepatitis $\mathrm{B}$ virus reactivation in occult viral carriers undergoing high-risk immunosuppressive therapy. Am J Gastroenterol. 2016;111:1788-95. doi:10.1038/ajg.2016. 436

25. Hsiao LT, Wang HY, Yang CF, et al. Human cytokine genetic variants associated with HBsAg reverse seroconversion in rituximab-treated non-Hodgkin lymphoma patients. Medicine (Baltimore) 2016;95:e3064 